\title{
Connotation and Denotation of the Chinese Medicine Dispensing Pharmacy
}

\author{
M.S.MIAO, S.TIAN \\ Henan University of Traditional Chinese Medicine, Henan, 450008, China
}

\begin{abstract}
The Chinese Medicine dispensing pharmacy is study the prescription of clinical medication review, deployment, monitoring, management, usage and other related knowledge of a discipline, its the important part of traditional Chinese medicine clinical applications. This paper discusses the Chinese Medicine dispensing pharmacy, aimed at correct defining the connotation and denotation of the the Chinese Medicine dispensing pharmacy, clear the direction of the Chinese Medicine dispensing pharmacy, and provide a reference for the modern study of the Chinese Medicine dispensing pharmacy.
\end{abstract}

KEYWORD: The Chinese Medicine dispensing pharmacy; combination; denotation

\section{INTRODUCTION}

The Chinese Medicine dispensing pharmacy refers to the relevant medical personnel according to the doctor's prescription or the needs of patients, and the pieces of chinese medicine or proprietary Chinese medicine is prepared in accordance with the relevant requirements of the operation, to provide patients with the use of the process, and is a liable technique. Its operation is a process of the prescription, pharmacology, the patient's condition and other factors considered, and it is one of the focus of the work of the hospital pharmacy. Its use operating techniques include trial side, pricing, deployment, review, packaging, and medicine and so on. The main content of the work is uniform the swap of the pieces of chinese medicine, norms the prescription name of pieces of chinese medicine(including the used name of doctors and manufacturers for the pieces of chinese medicine), study the dose-effect relationship of the pieces of chinese medicine, etc. And its an important part of traditional Chinese medicine clinical applications, and its a discipline under the guidance of the theory of Chinese medicine, research related to knowledge and technology. This paper discusses the connotation and denotation of the Chinese Medicine dispensing pharmacy.

\section{THE ORIGIN OF THE CHINESE MEDICINE DISPENSING}

TCM originated in primitive society, has a long history, its a experience product of working people in the long-term fight against the disease. The origin and development of the Chinese Medicine dispensing is also underwent a long process of practice. According to records, the Shang Dynasty created a decoction, and has the "soup liquid by Law", the flags of the decoction widespread use is the emergence of the pieces of chinese medicine recipes[1], is the earliest literature recorded dispensing. The spring and autumn period and the warring states period of "Yellow Emperor", summarizes the theory of compatibility, and recorded a simple prescription, its appears for the formation of the theory of traditional Chinese medicine dispensing laid the theoretical foundation. The Western Han Dynasty in the "Shen Nong's herbal classic" clearly documented the theory and operation of dispensing in order, for example, records about the time of taking medicine, "Disease in mediastinoscopy or more, first eat food and then taking medication. Disease in the confidant less, taking medication first and then eat. Disease in the limbs, blood who should fasting while in day. Disease in the bone marrow were advised full and in the night," etc. It appears to provide a theoretical guidance for Chinese medicine dispensing, marking the formation of the theory of the Chinese Medicine dispensing[2].

Changsha Mawangdui unearthed "Fifty-two diseases party "total contains 283 medical side, not only the number of compound is, and the dosage is varied, both orally and topical, bathing, and topical formulations and so on, which basised for the formation of dispensing technical. The Eastern Han 
Dynasty Zhang Zhonging's "Treatise on Febrile Diseases", it has records decoction, powders, pills, as well as wine, pastes and other formulations, and gave a detailed description of the dispensing method for a variety of formulations, in addition to also made a presentation about the furnace and methods of tisane, the methods of taking medication, contraindications and so on, marking the form of the Chinese medicine dispensing technology operations, such as lilies washing to flooding, body wash; sulfur casual side, powder and to fire smoke, etc. Liang Dynasty "Herbal Classic variorum," has a detailed description of the theory of the Chinese medicine dispensing, medicinal metrology and other content, standardized the weighing standard of the Chinese medicine dispensing. Tang Dynasty "emergency preparedness Qian Jin Yi Fang", not only summarizes the relevant contents of the previous dispensing, and recorded the dispensing necessary tools such as knives, scales, bucket, iron mortar, jade hammer, magnetic bowl, etc.

"Newly Revised Materia Medica" as the first China's pharmacopoeia of herbal medicine, standardized the methods of the Chinese Medicine dispensing in the nationwide, which greatly promoted the development of the Chinese Medicine dispensing."Tang Code" as a more comprehensive law in ancient China, it also has made the appropriate provisions of the Chinese Medicine dispensing. Stressed the dispensing medicine should be consistent with prescription, the doctor should be subject to appropriate punishment if has the error.

\section{CONNOTATION OF THE CHINESE MEDICINE DISPENSING}

\subsection{The operation of the Chinese medicine dispensing}

The operation of the Chinese medicine dispensing including trial square meter, dispensing weighing, weight, special treatment, review and other steps, its an important part which related to the clinical efficacy and safety of drugs. Prescription is the basis of the Chinese Medicine dispensing, the recipient who received a prescription, the prescription should be reviewed in accordance with the order of the various projects, such as the relationship between age and drug dose, has or not repeat medicine in prescription, has or not excessive of the toxicity and potent medicines, has or not "Eighteen anti" and "Nineteen fear" of drugs and require special decoction of drugs,etc. The basic method of dispensing commonly used is diminishing method, according the prescription order from top to bottom, left to right weighed, the total weighed amount and pay per volume to scale according the decreasing method accurately weighed, so that a scales a drug. When dispensing to see the prescription at any time, in order to avoid leakage dispensing, mismatch, requiring weight per dose error should be within \pm $5 \%$. To correct use the measuring instruments, such as Dengzi scales, electronic scales, days of equality, inspected once a year, in order to improve efficiency. When dispensing and down drugs, generally when there has leafy in prescription, to pour in a central location, the other pieces of medicine should put into a slash from the the right corner of paper to lower right corner. If it has flowers herbs, it will have to fall on the grass. If has the cutting big pieces of medicine, then pour in the corners. If there has medicine need to another package should be aside, indicate the decoction method and the taking method, in order to review. Review is an important part to ensure that the safety of patient take the medication and the quality of the recipe. When reviewing check the taste, the quality of medicines, component, whether has the instructions that drugs require special handling and so on, after the review to written the patient's name on the bag. The drug should be dispensing by patient name, check with the supplied product is complete, to account for the patient the decoction method, medication contraindications and other specific content.

\subsection{The theory of the Chinese Medicine dispensing}

The Chinese Medicine dispensing is in the guidance of Chinese medicine theory, carried out a legally responsible technical operations, and therefore require medicine dispensing staff to have some knowledge of Chinese medicine theory. Learn the basics knowledge of traditional Chinese medicinal, learn the taste and indications medicine, master different drugs of the four properties like cold, hot, warm, cool and functional characteristics. And also learn commonly used prescription of composition drugs in the clinical and efficacy characteristics, such as ephedra soup, SanJuYin, Sijunzitang, and so on, grasp the contents of commonly used prescriptions, in order to apply at the time of the trial swap party. In addition to meeting the needs of the development of modern society, the dispensing industry also has compilation a series of the Chinese Medicine dispensing books, such as "Clinical practical Chinese medicine dispensing technology", "Chinese medicine dispensing with maintenance", "Chinese medicine prescription and dispensing specification", "Chinese medicine dispensing entry" and so on, and rich content related to the dispensing according to modern scientific theories and techniques.

\subsection{The relevant laws and regulations of the Chinese Medicine dispensing}

The Chinese Medicine dispensing work is a rigorous 
and complex professional, highly technical work, the quality of dispensing is good or bad, directly related to medicine safety, clinical efficacy and the safety of the patients life, its also an important part to ensure the medicine are safe, effective, to achieve reasonable clinical medication. Therefore, the state has the interpretation of the Chinese medicine dispensing in "Chinese Pharmacopoeia", "drug management measures", "Traditional Chinese Medicine processing specifications," "Drug Standards" and other laws and regulations. According to these regulations, developed a management system of the Chinese Medicine dispensing, such as the Chinese Medicine dispensing working system, prescription management system, special medicine dispensing and management and so on, such as pharmacists are not in accordance with the stipulations dispensing the prescription drugs of narcotic, psychotropic, causing serious consequences, according to the article 56th of the "Prescription Management" regulations, the original issuing department shall revoke the practicing certificate.2009 the State Council issued the "hospital pharmacy basic standards", it provide a solid guarantee policy for the development of the pharmaceutical industry, also makes the Chinese Medicine dispensing gradually standardized and scientific.

\section{DENOTATION OF THE CHINESE MEDICINE DISPENSING}

The denotation of the discipline should be able to reactions a wide range that involved in its connotation, its relative with the connotation, and has a close relationship[3].On the view of the connotation of the Chinese Medicine dispensing, the denotation of the Chinese Medicine dispensing mainly relates to Traditional Chinese medicine clinical pharmacy, the science of Chinese medicine processing, the science of Chinese Medicine identification, the science of traditional Chinese medicine conservation, the science of pharmacology of traditional Chinese medical formulas, the science of Chinese medicine pharmacology and other subjects. So we should use modern science technology and methods, in-depth exploration the denotation of the Chinese Medicine dispensing, development the Chinese Medicine dispensing related disciplines, improve the pharmaceutist participate in clinical work, help physicians enhance the level of use drugs and the quality of medical, and provide the level of drug counseling services for physicians, nurses, patients and customers, making the drug works best effect.

\subsection{Traditional Chinese medicine clinical pharmacy}

Traditional Chinese medicine clinical pharmacy is an important branch of Clinical Pharmacy, its an applied discipline under the guidance of the theory of Chinese medicine, take the patients as the objects, by the rational drug use in traditional Chinese medicine for research priorities, studies the basic theory, the safety and effectiveness of clinical use, and application regular. The Chinese Medicine dispensing as an important part of the clinical application of traditional Chinese medicine, the quality of dispensing direct impact the level of Chinese medicine clinical pharmacy services. In turn, the development of the traditional Chinese medicine clinical pharmacy will certainly lead to the development and research of the Chinese Medicine dispensing.

\subsection{The science of Chinese medicine processing}

The science of Chinese medicine processing is a discipline specializing in the theory of Chinese medicine processing, technology, specifications, quality standards, history and development. The task of the traditional Chinese medicine processing is to make the medicine according to the body and illness patients, using different processing method, in order to achieve the purpose of attenuated efficienc, so as to ensure the effectiveness and safety of clinical medication, to give full play the role of traditional Chinese medicine to prevent and cure diseases. The traditional Chinese medicine can be said to be the foundation of traditional Chinese medicine, only processing medicinal up to standard, adjust personnel can correct transfers, in order to better services for patients, improve the quality of clinical medicine.

\subsection{The science of Chinese Medicine identification}

The science of Chinese Medicine identification is a application discipline to identify and study the variety and quality of traditional Chinese medicine, formulated Chinese medicine quality standards, find and expand the source of a new medicine. The main research content is traditional Chinese medicine and base of the original identification, including identification methods and identification standards. Accurate identification of traditional Chinese medicine is a fundamental part in the development of the pharmaceutical industry, and concern. Pharmaceutist master these identification capability, accurately identify the authenticity and quality of pros and cons of traditional Chinese medicines, so as to ensure the quality of clinical use, ensure that the clinical efficacy of Chinese medicine. As species confusion, pharmaceutist should be able to identify on the basis of the application of traditional 
experience, combined with modern identification technology, to ensure the quality of traditional Chinese medicine from the source, so as to achieve the purpose of the drug to the disease.

\subsection{The science of traditional Chinese medicine conservation}

The science of traditional Chinese medicine conservation is a comprehensive application discipline study the natural property of various commodities traditional Chinese and the deterioration regular influence by outside, through the scientific methods and protective measures to safeguard the security of the storage product. It provides a theoretical basis for the traditional Chinese medicine, the pieces of Chinese medicine and preparations of goods in quality assurance during transport and storage. Due to the increasing demand for Chinese herbal medicine, and its circulation has become even more frequent, but traditional Chinese medicine as a special goods, save it improper, it will lead to decreased efficacy or failure, thus affecting the clinical efficacy, make the Chinese herbal medicine resources wasted. So development and study the science of traditional Chinese medicine conservation, guarantee the quality of traditional Chinese medicine, to better promote the development of the Chinese Medicine dispensing, rich the theoretical connotation of the Chinese medicine dispensing.

\subsection{The science of pharmacology of traditional Chinese medical formulas}

The science of pharmacology of traditional Chinese medical formulas is a discipline study the governing law and the law of prescription compatibility and its clinical use, prescription is an important part of the science of pharmacology of traditional Chinese medical formulas research. Medicine as the main content of prescription, each of are made one or more medicines in accordance with certain compatibility theoretical dispensing and formed. Because prescription is reasonable compatibility doctor according to the patient's condition, if the dispensing personnel do not have the prescriptions of the basic theory, it is difficult to understand the compatibility of prescription medicines and indications, its not good to judge the priorities of the patient's condition, thus do the accuracy of the dispensing medicines and achieve the accuracy of judgment the prescription medicines, proper patient medication guide, to improve the effectiveness of medication.

\subsection{The science of Chinese medicine pharmacology}

The science of Chinese medicine pharmacology is a discipline based on the theory of Chinese medicine, using the method of modern pharmacology experiment, study the interaction between traditional Chinese medicine and the body and its mechanism, clarify the principle for its disease prevention, and develop the modern Chinese medicine and the innovation of Chinese medicine, create a modern property theory of traditional Chinese medicine. Traditional Chinese medicine prescription is based on the "monarch, minister, assistant, make" principle, the dose of the prescription medicine is not the same, its pharmacological effects are not the same, therefore prescription dispensing is accurate or not, directly related to the pharmacological action played. Since ancient times, it has the said "Chinese do not pass the secret on to say the amount", shows that the importance of dose, modern pharmacological studies have confirmed the therapeutic effects and indications are closely related the dosage of medicine. Therefore, the dispensing personnel should carefully and correct dispensing, ensure the pharmacological effects play. In addition, the research and development of traditional Chinese medicine pharmacology swap will promotion the further explore of the Chinese Medicine dispensing.

\section{CONCLUSIONS}

The Chinese Medicine dispensing is a highly technical discipline, the quality of dispensing closely related to the safety and effectiveness of clinical medication, dispensing is correct or not directly affect the efficacy and the patient's condition, rehabilitation and life safety. In addition, a reasonable dispensing also favor the formation of the concept of rational drug use, make the dispensing personnel to form the "minor ailments when a doctor, a serious illness when the staff," sense of responsibility, do a good job with the consultant, ensure the reasonable, safe, and effective in patients with medication. But in the course of the dispensing clinical medicine, there still has some problems directly affect the effectiveness of clinical medicine, such prescriptions are not standardized, so that the dispensing personnel can not correctly identify the name of medicine, resulting in changes in the efficacy of prescription; the dispensing personnel lack of knowledge likes Chinese medicine processing and Chinese Medicine identification and so on, makes some non-genuine or counterfeit goods mixed in, make the patients with medication has some security risks, and so on. Dispensing medicine plays a vital role in the treatment of traditional Chinese medicine, combining the Chinese Medicine dispensing and modern information technology, such as the use of electronic prescribing, automatic packaging machines, automatic dispensing machines and so on, in order to increase the accuracy and 
efficiency of dispensing. The Chinese Medicine dispensing as an important part of the traditional Chinese medicine clinical pharmacy, and with a combination of the traditional Chinese medicine clinical pharmacy, guide rational use of clinical medicine, in order to better serve for the clinical medicine. In addition, standardized the operation of the dispensing personnel, improve the professional quality of the dispensing personnel, it also ensure the safety of medicine, improve the therapeutic effect of medicine. Scientific definition the connotation and denotation of the Chinese Medicine dispensing, dig its resources, promote the development of its related disciplines, thereby indicating the direction of future research and development of the Chinese Medicine dispensing, in order to has a better clinical services of traditional Chinese medicine.

\section{REFERENCES}

[1] Huihua Hu, DanYang,Liu, Yunhua,Chen 2013. Historical Evolution and Development of Traditional Chinese Medicine Dispensing. Pharmaceutical Monographs 22(19):1-4.

[2] Xuemin Zhao, Xiaojuan Zhang, Huaqiang Zhai. 2014. Briefly analysis on academic origins of traditional Chinese medicine dispensing. China Journal of Chinese Materia Medica 39 (8):1530-1533.

[3] Jin Teng. 2013. Define and study reflect encephalopathology connotation and denotation. JETCM22 (6):918,958. 